\title{
Representation of the Signal Transduction with Aberrance Using Ipi Calculus ${ }^{\star}$
}

\author{
Zhang, M. ${ }^{\star \star 1}, \mathrm{Li}, \mathrm{G} .{ }^{1}, \mathrm{Fu}, \mathrm{Y}^{1},{ }^{1}$ Zhang, $\mathrm{Z}^{2}{ }^{2}$, and He, L. ${ }^{2}$ \\ 1 BASICS, Department of Computer Science and Engineering, \\ Shanghai Jiao Tong University, Shanghai 200030, China \\ \{zhangmin, liguoqiang, yxfu\}@sjtu.edu.cn \\ 2 BDCC, College of Life Science and Biotechnology, \\ Shanghai Jiao Tong University, Shanghai 200030, China \\ \{zhangzz, helin\}@sjtu.edu.cn
}

\begin{abstract}
The pi calculus has been applied to modelling biochemical networks. In these applications, the modelling is done without considerations to exceptions. The Ipi calculus, the Interference pi calculus, is introduced to describe the signal transduction with aberrance. The calculus is obtained by adding two aberrant actions into the pi calculus and a tag system to check existing aberrance. A model of the signal transduction, RTK-MAPK, with the aberrant Ras is highlighted to illustrate the expressive power of the Ipi calculus.
\end{abstract}

\section{Introduction}

In recent years, various approaches from computer science have been adapted for the research of biochemical processes. These include boolean networks, petri nets, and object-oriented databases, to name a few. The pi calculus $[1,6]$ is an alternative way to model biochemical processes. In the pi calculus approach, molecules and their individual domains are treated as computational processes, where their complementary structural and chemical determinants correspond to the communication channels. Chemical interaction and subsequent modification coincide with communication and channel transmission. There are some related research about modelling various biochemical systems based on the pi calculus. Such systems cover for instance the signal transduction (ST for short) $[4,5,2$, 3]. However the biochemical systems considered so far are restricted in the sense that one assumes that there are no exceptions when they evolve.

ST, a process linking the detection of certain kinds of external events to biochemical responses on the part of the cell is a very important biochemical process in biology. An aberrant ST is the cause of many diseases challenged by modern medicine, including cancer, inflammatory diseases, cardiovascular disease and neuropsychiatric disorders. In the search for treatments, cures and preventions

\footnotetext{
* The work is supported by the Young Scientist Research Fund (60225012) and BDCC (03DZ14025).

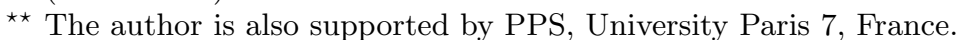


of these diseases, in-depth understanding of the biochemical processes of ST is crucial.

In order to describe more complex biochemical systems like the aberrant ST, we develop a calculus called the Interference pi calculus by extending the pi calculus. The calculus is obtained by adding two aberrant actions into the pi calculus and a tag system to check existing aberrance. We also illustrate our system using a model of the ST, RTK-MAPK, with the aberrant Ras (a signal that can lead to cancer) which will result to pathological changes.

In this short paper, we have to assume that the reader is familiar with the pi calculus and has some knowledge of biochemical systems.

\section{The Interference Pi Calculus}

In this section we define the syntax and the semantics of the Interference pi calculus.

\subsection{Syntax}

Processes evolve by performing actions. In process algebra action capabilities are introduced by prefix capabilities. In our calculus we define prefix as a pair, where each capability has a label we call interference coefficient, regulating whether it can act in a normal way, or in an aberrant manner. So we define our interference coefficient primitive before we introduce the prefix.

We assume an infinite countable set $\mathcal{N}$ of names and an infinite countable set $\mathcal{V}$ of values. We let $x, y, \ldots$ range over the names. Let $\sigma, \rho$ be functions from $\mathcal{N}$ to $\mathcal{V}$. One can think of $\sigma$ as an interference function and that $\sigma(x)$ as the interference degree of $x$. The function $\rho$ is a critical function and that $\rho(x)$ is the critical value of the interference degree of $x$. The interference coefficient can be defined below:

Definition 1 (Interference Coefficient). For $x \in \mathcal{N}$, let $i_{x}$ be $|\rho(x)-\sigma(x)|$. We say that $i_{x}$ is the interference coefficient of $x$.

Intuitively, when $i_{x}$ is equal to zero, we take that $x$ is in an aberrant state; when $i_{x}$ is not zero, we think that $x$ is still in a normal state. For convenience of representation, when $i_{x}$ is equal to zero, we write 0 as the interference coefficient of $x$. Otherwise we write $i_{x}$ as the interference coefficient of $x$.

We also define two symbols, $\S$ and $\sharp$, to represent the aberrance capability. Here $\S$ represents the killer capability and $\sharp$ the propagation capability. When a process has the killer capability, it will terminate immediately. And when a process has the propagation capability, it will duplicate himself infinitely.

We define the prefix of Ipi calculus as follows:

Definition 2 (Prefix).

$$
\begin{gathered}
\left\langle i_{\pi}, \pi\right\rangle:=\left\langle i_{x}, \bar{x}(y)\right\rangle\left|\left\langle i_{x}, x(y)\right\rangle\right|\left\langle i_{x}, \bar{x}\right\rangle \mid\left\langle i_{x}, x\right\rangle \\
\pi_{i}:=\left\langle i_{\pi}, \pi\right\rangle\left|\langle 0, \S\rangle \pi_{i}\right|\langle 0, \sharp\rangle \pi_{i}
\end{gathered}
$$


The capabilities of $\left\langle i_{\pi}, \pi\right\rangle$ is the same as in the $\pi$-calculus. Here are some explanations of the $\pi_{i}$ capabilities: $\langle 0, \S\rangle$ has the capability that kills all the processes; $\langle 0, \sharp\rangle$ has the capability that duplicates processes infinitely; $\langle 0, \S\rangle \pi_{i}$ and $\langle 0, \sharp\rangle \pi_{i}$ are the substitution capabilities: they are respectively the capabilities $\langle 0, \S\rangle$ and $\langle 0, \sharp\rangle$ if the interference coefficient $i_{\pi}$ of the $\pi$ is zero.

We now define the processes of the Ipi calculus. The expression of a process is also a pair $\left\langle I_{P}, P\right\rangle$ where $I_{P}$ is the tag of the process $P$. Using the tag in processes we can know the existence of aberrance. If $0 \in I_{P}$ we say that $P$ has aberrance. If for any $i \in I_{P}$ one has that $i \neq 0$ then we say that $P$ is normal. The value of tag is produced recursively.

Definition 3 (Process). The Ipi processes are defined as follows:

$$
\begin{aligned}
\left\langle I_{P}, P\right\rangle:= & \left\langle I_{0}, 0\right\rangle\left|\pi_{i} \cdot\left\langle I_{P}, P\right\rangle\right| \pi_{i} \cdot\left\langle I_{P}, P\right\rangle+\pi_{i}^{\prime} \cdot\left\langle I_{P^{\prime}}, P^{\prime}\right\rangle\left|\left\langle I_{P}, P\right\rangle\right|\left\langle I_{P^{\prime}}, P^{\prime}\right\rangle \mid \\
& (\nu x)\left\langle I_{P}, P\right\rangle \mid\left\langle I_{P}, P\right\rangle ;\left\langle I_{P^{\prime}}, P^{\prime}\right\rangle
\end{aligned}
$$

The syntax of the tags are defined inductively by the following rules, where the

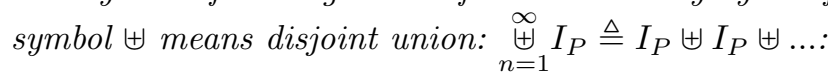

$$
\begin{aligned}
& \overline{I_{0}=\emptyset} \quad \text { o- } t \quad \frac{\left\langle I_{P}, P\right\rangle=\left\langle i_{\pi}, \pi\right\rangle \cdot\left\langle I_{Q}, Q\right\rangle}{I_{P}=\left\{i_{\pi}\right\} \uplus I_{Q}} t-N \\
& \frac{\left\langle I_{P}, P\right\rangle=\langle 0, \S\rangle\left\langle i_{\pi}, \pi\right\rangle \cdot\left\langle I_{Q}, Q\right\rangle}{I_{P}=\{0\}} \S-t \quad \frac{\left\langle I_{P}, P\right\rangle=\langle 0, \sharp\rangle\left\langle i_{\pi}, \pi\right\rangle \cdot\left\langle I_{Q}, Q\right\rangle}{I_{P}=\underset{n=1}{\uplus}\left(\{0\} \uplus\left\{i_{\pi}\right\} \uplus I_{Q}\right)} \sharp-t \\
& \frac{\left\langle I_{P}, P\right\rangle=\left\langle i_{\pi_{i}}, \pi_{i}\right\rangle \cdot\left\langle I_{Q}, Q\right\rangle+\left\langle i_{\pi_{i}^{\prime}}, \pi_{i}^{\prime}\right\rangle \cdot\left\langle I_{R}, R\right\rangle}{I_{P}=f\left\langle\left\{i_{\pi_{i}}\right\} \uplus I_{Q},\left\{i_{\pi_{i}^{\prime}}\right\} \uplus I_{R}\right\rangle} \text { sum- } t \\
& \frac{\left\langle I_{P}, P\right\rangle=\left\langle I_{Q}, Q\right\rangle \mid\left\langle I_{R}, R\right\rangle}{I_{P}=I_{Q} \cup I_{R}} \text { com- } t \quad \frac{\left\langle I_{P}, P\right\rangle=(\nu x)\left\langle I_{Q}, Q\right\rangle}{I_{P}=I_{Q}} \text { res- } t \\
& \frac{\left\langle I_{P}, P\right\rangle=\left\langle I_{Q}, Q\right\rangle ;\left\langle I_{R}, R\right\rangle}{I_{P}=I_{Q} \uplus I_{R}} \text { seq- } t
\end{aligned}
$$

In the above definition, $\left\langle I_{P}, I_{Q}\right\rangle$ is a pair, $f$ is the projection, and $f_{P, Q}\left\langle I_{P}, I_{Q}\right\rangle$ represents the tag of the process which has the operator "sum". $I_{P}$ and $I_{Q}$ are nondeterministically chosen as the process $P$ or $Q$ is chosen to act.

Intuitively the constructs of the Ipi processes have the following meaning: $\left\langle I_{0}, 0\right\rangle$ is the inert process. The prefix process $\pi_{i} \cdot\left\langle I_{P}, P\right\rangle$ has a single capability imposed by $\pi_{i}$, that is, the process $\left\langle I_{P}, P\right\rangle$ cannot proceed until that capability has been exercised. The capabilities of the sum $\pi_{i} \cdot\left\langle I_{P}, P\right\rangle+\pi_{i}^{\prime} \cdot\left\langle I_{P^{\prime}}, P^{\prime}\right\rangle$ are those of $\pi_{i} \cdot\left\langle I_{P}, P\right\rangle$ plus those of $\pi_{i}^{\prime} \cdot\left\langle I_{P^{\prime}}, P^{\prime}\right\rangle$. When a sum exercises one of its capabilities, the other is rendered void. In the composition process $\left\langle I_{P}, P\right\rangle \mid\left\langle I_{Q}, Q\right\rangle$, the components $\left\langle I_{P}, P\right\rangle$ and $\left\langle I_{Q}, Q\right\rangle$ can proceed independently and can interact via shared channels. In the restriction process $(\nu x)\left\langle I_{P}, P\right\rangle$, the scope of the name $x$ is restricted to $\left\langle I_{P}, P\right\rangle$. The sequential process $\left\langle I_{P}, P\right\rangle ;\left\langle I_{P^{\prime}}, P^{\prime}\right\rangle$ can run the process $\left\langle I_{P^{\prime}}, P^{\prime}\right\rangle$ after the process $\left\langle I_{P}, P\right\rangle$. 


\subsection{Semantics}

The structural congruence $\equiv$ is the least equivalence relation on processes that satisfies the following equalities:

$$
\begin{aligned}
\left\langle I_{P}, P\right\rangle \mid\left\langle I_{Q}, Q\right\rangle & \equiv\left\langle I_{Q}, Q\right\rangle \mid\left\langle I_{P}, P\right\rangle \\
\left(\left\langle I_{P}, P\right\rangle \mid\left\langle I_{Q}, Q\right\rangle\right) \mid\left\langle I_{R}, R\right\rangle & \equiv\left\langle I_{P}, P\right\rangle \mid\left(\left\langle I_{Q}, Q\right\rangle \mid\left\langle I_{R}, R\right\rangle\right) \\
\left\langle I_{P}, P\right\rangle+\left\langle I_{Q}, Q\right\rangle & \equiv\left\langle I_{Q}, Q\right\rangle+\left\langle I_{P}, P\right\rangle \\
\left(\left\langle I_{P}, P\right\rangle+\left\langle I_{Q}, Q\right\rangle\right)+\left\langle I_{R}, R\right\rangle & \equiv\left\langle I_{P}, P\right\rangle+\left(\left\langle I_{Q}, Q\right\rangle+\left\langle I_{R}, R\right\rangle\right) \\
(\nu x)\left\langle I_{0}, 0\right\rangle & \equiv\left\langle I_{0}, 0\right\rangle \\
(\nu x)(\nu y)\left\langle I_{P}, P\right\rangle & \equiv(\nu y)(\nu x)\left\langle I_{P}, P\right\rangle \\
\left((\nu x)\left\langle I_{P}, P\right\rangle\right) \mid\left\langle I_{Q}, Q\right\rangle & \equiv(\nu x)\left(\left\langle I_{P}, P\right\rangle \mid\left\langle I_{Q}, Q\right\rangle\right) \text { if } x \notin F N(Q)
\end{aligned}
$$

Let $I_{P}, I_{Q}$ be the tags of the processes $P$ and $Q$. We define

$$
I_{P}=I_{Q} \Leftrightarrow\left\langle I_{P}, P\right\rangle \equiv\left\langle I_{Q}, Q\right\rangle
$$

So we have defined an equivalence on the tags in terms of the structural equivalence.

The reaction relation, introduced initially by Milner [1], is a concise account of computation in the pi calculus. A reaction step arises from the interaction of the adjacent process with $\bar{m}\langle M\rangle . P$ and $m(x) . Q$, which is also included in the Ipi calculus. Besides this rule, our reaction relation also include two new rules representing reactions with aberrance. All the rules react with their tags reacting simultaneously. We define them and their tag reaction rules blow:

$$
\begin{aligned}
& \overline{\langle 0, \S\rangle\left\langle i_{\pi}, \pi\right\rangle \cdot\left\langle I_{P}, P\right\rangle \longrightarrow\langle\emptyset, 0\rangle} ; \quad \overline{\{0\} \backslash\{0\}=\emptyset}{ }^{\text {pre- } \S} ; \\
& \overline{\langle 0, \sharp\rangle\left\langle i_{\pi}, \pi\right\rangle \cdot\left\langle I_{P}, P\right\rangle \longrightarrow\left\langle i_{\pi}, \pi\right\rangle \cdot\left\langle I_{P}, P\right\rangle ;\langle 0, \sharp\rangle\left\langle i_{\pi}, \pi\right\rangle \cdot\left\langle I_{P}, P\right\rangle} ;
\end{aligned}
$$

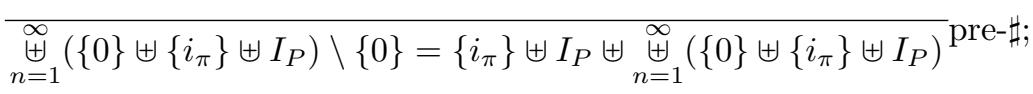

$$
\begin{aligned}
& \overline{\left\langle i_{x}, \bar{x}(z)\right\rangle \cdot\left\langle I_{Q}, Q\right\rangle\left|\left\langle i_{x}, x(y)\right\rangle \cdot\left\langle I_{P}, P\right\rangle \longrightarrow\left\langle I_{Q}, Q\right\rangle\right|\left\langle I_{P}, P\right\rangle\{z / y\}} ; \\
& \overline{\left(\left\{i_{x}\right\} \uplus I_{Q}\right) \cup\left(\left\{i_{x}\right\} \uplus I_{P}\right) \backslash\left\{i_{x}\right\}=I_{Q} \cup I_{P}} \text { com-N; } \\
& \frac{\left\langle I_{P}, P\right\rangle \longrightarrow\left\langle I_{P^{\prime}}, P^{\prime}\right\rangle}{\left\langle I_{P}, P\right\rangle+\left\langle I_{Q}, Q\right\rangle \longrightarrow\left\langle I_{P^{\prime}}, P^{\prime}\right\rangle} ; \quad \frac{I_{P} \backslash\left\{i_{y}\right\}=I_{P^{\prime}}}{f_{P}\left\langle I_{P}, I_{Q}\right\rangle \backslash\left\{i_{y}\right\}=I_{P^{\prime}}} ; \\
& \frac{\left\langle I_{P}, P\right\rangle \longrightarrow\left\langle I_{P^{\prime}}, P^{\prime}\right\rangle}{\left\langle I_{P}, P\right\rangle\left|\left\langle I_{Q}, Q\right\rangle \longrightarrow\left\langle I_{P^{\prime}}, P^{\prime}\right\rangle\right|\left\langle I_{Q}, Q\right\rangle} ; \quad \frac{I_{P} \backslash\left\{i_{y}\right\}=I_{P^{\prime}}}{I_{P} \cup I_{Q} \backslash\left\{i_{y}\right\}=I_{P^{\prime}} \cup I_{Q}} ; \\
& \begin{array}{ll}
\left\langle I_{P}, P\right\rangle \longrightarrow\left\langle I_{P^{\prime}}, P^{\prime}\right\rangle \quad x \neq y \\
(\nu x)\left\langle I_{P}, P\right\rangle \longrightarrow(\nu x)\left\langle I_{P^{\prime}}, P^{\prime}\right\rangle
\end{array} ; \quad \frac{I_{P} \backslash\left\{i_{y}\right\}=I_{P^{\prime}}}{I_{P} \backslash\left\{i_{y}\right\}=I_{P^{\prime}}} ; \\
& \frac{\left\langle I_{Q}, Q\right\rangle \equiv\left\langle I_{P}, P\right\rangle \quad\left\langle I_{P}, P\right\rangle \longrightarrow\left\langle I_{P^{\prime}}, P^{\prime}\right\rangle \quad\left\langle I_{P^{\prime}}, P^{\prime}\right\rangle \equiv\left\langle I_{Q^{\prime}}, Q^{\prime}\right\rangle}{\left\langle I_{Q}, Q\right\rangle \longrightarrow\left\langle I_{Q^{\prime}}, Q^{\prime}\right\rangle} ; \\
& \frac{I_{Q}=I_{P} \quad I_{P} \backslash\left\{i_{x}\right\}=I_{P^{\prime}} \quad I_{P^{\prime}}=I_{Q^{\prime}}}{I_{Q} \backslash\left\{i_{x}\right\}=I_{Q^{\prime}}} .
\end{aligned}
$$


The first and the second rules deal with reactions with aberrance: the former says that the resulting process is terminated and its tag also changes to empty set. The latter declares that the resulting process duplicates itself infinitely and its tag also duplicates itself. The third reaction rule deals with the interaction in which one sends a message with a channel while the other receives a message with the same channel so that they have an interactive action. This is quite common in the pi calculus. Each of the reduction rules also includes its tag reductions, which means that all the reaction rules are closed in the summation, composition, restriction and structural congruence.

\section{An Example in ST Pathway with the Aberrance}

In this section we take a look at an example of ST pathways using the new calculus.

\subsection{The RTK-MAPK pathway}

In biology pathways of molecule interactions provide communication between the cell membrane and intracellular endpoints, leading to some change in the cell.

We focus our attention on the well-studied RTK-MAPK pathway. The RTKMAPK pathway is composed of 14 kinds of proteins. A protein ligand molecule (GF), with two identical domains, binds two receptor tyrosine kinase (RTK) molecules on their extracellular part. The bound receptors form a dimeric complex, cross-phosphorylate and activate the protein tyrosine kinase in their intracellular part. The activated receptor can phosphorylate various targets, including its own tyrosines. The phosphorylated tyrosine is identified and bound by an adaptor molecule SHC. A series of protein-protein binding events follows, leading to formation of a protein complex (SHC, GRB2, SOS, and Ras) at the receptor intracellular side. Within this complex the SOS protein activates the Ras protein, which in turn recruits the serine/threonine protein kinase, Raf, to the membrane, where it is subsequently phosphorylated and activated. A cascade of phosphorylations/activations follows, from Raf to MEK1 to ERK1. This cascade culminates in the activation of the threonine and tyrosine protein kinase, ERK1. Activated ERK1 translocates to the nucleus, where it phosphorylates and activates transcription factors.

Within the framework of Ipi calculus, we set some principles for the correspondence. Firstly, we choose the functional signaling domain as our primitive process. This captures the functional and structural independence of domains in signaling molecules. Secondly, we model the component residues of domains as communication channels that construct a process. Finally, molecular interaction and modification is modelled as communication and the subsequent change of channel names. 


\subsection{Representation for the Ras and the Aberrant Ras}

Fig.1 gives an example Ras Activation of the ST pathway, RTK-MAPK. In this ST, Ras Activation is the part of the pathway. At the normal state, the protein-to-protein interactions bring the SOS protein close to the membrane, where Ras can be activated. SOS activates Ras by exchanging Ras's GDP with GTP. Active Ras interacts with the first kinase in the MAPK cascade, Raf. GAP inactivates it by the reverse reaction. Aviv Regev and his colleagues have given the representation of normal RTK-MAPK using the pi calculus.

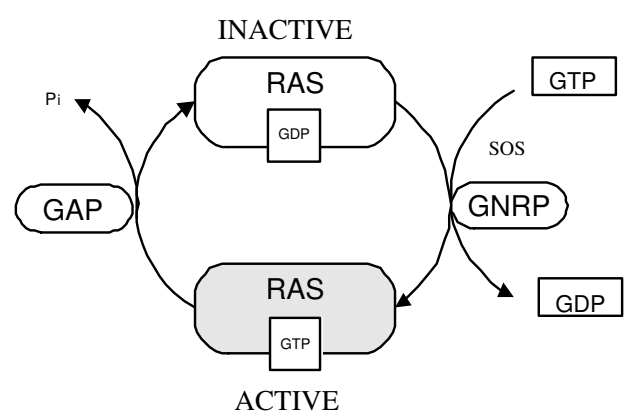

Fig.1. Ras Activation

The interpretation of Ras in the Ipi calculus can be done in the following manner:

The system defined in (1) is a collection of concurrently operating molecules, seen as processes with potential behavior. Here the operator $\mid$ is the concurrent combinator:

$$
\begin{aligned}
\left\langle I_{S}, S Y S T E M\right\rangle::= & \left\langle I_{R S}, R A S\right\rangle\left|\left\langle I_{S}, S O S\right\rangle\right|\left\langle I_{G}, G A P\right\rangle \mid \\
& \left\langle I_{R F}, R A F\right\rangle \mid \cdots
\end{aligned}
$$

A protein molecule is composed of several domains, each of which is modelled as a process as well. In (2) through (5) the detailed Ipi calculus programs for the proteins Ras, SOS, Raf and GAP are given:

$$
\begin{aligned}
\left\langle I_{R S}, R A S\right\rangle::= & \left\langle I_{I S I}, I N A S W I_{-} I\right\rangle \mid\left\langle I_{I S I I}, I N A S W I_{-} I I\right\rangle \\
\left\langle I_{S}, S O S\right\rangle::= & \left\langle I_{S S B}, S_{-} S H 3_{-} B S\right\rangle \mid\left\langle I_{S G}, S_{-} G N E F\right\rangle \\
\left\langle I_{R F}, R A F\right\rangle::= & \left\langle I_{R N}, R_{-} N t\right\rangle\left|\left\langle I_{R A C B}, R_{-} A C T_{-} B S\right\rangle\right|\left\langle I_{R M B}, R_{-} M_{-} B S\right\rangle \\
& \left|\left\langle I_{I R C}, I N A_{-} R_{-} C t\right\rangle\right|\left\langle I_{R A T B}, R_{-} A T P \_B S\right\rangle \\
\left\langle I_{G}, G A P\right\rangle::= & \left\langle i_{s}, \text { sg }\right\rangle\left(c_{-} \text {ras }\right) .\left\langle i_{c}, \overline{c_{-} \text {ras }}\right\rangle(g d p) .\left\langle I_{G}, G A P\right\rangle
\end{aligned}
$$

The molecules (or domains) interact with each other based on their structural and chemical complementarity. Interaction is accomplished by the motifs and residues that constitute a domain. These are viewed as channels or communica- 
tion ports of the molecule:

$$
\begin{aligned}
\left\langle I_{I S I}, I N A S W I_{-} I\right\rangle::= & \left\langle i_{b}, \overline{b b o n e}\right\rangle \cdot\left\langle I_{A S I}, A C T S W I_{-} I\right\rangle \\
\left\langle I_{I S I I}, I N A S W I_{-} I I\right\rangle::= & \left\langle i_{s g}, \overline{s g}\right\rangle\left(r s_{-} 1\right) \cdot\left\langle i_{r s 1}, r s_{-} 1\right\rangle(x) \cdot\left\langle i_{b}, b b o n e\right\rangle . \\
& \left\langle I_{A S I I}, A C T S W I_{-} I I\right\rangle \\
\left\langle I_{S G}, S_{-} G N E F\right\rangle::= & \left\langle i_{b}, b b o n e\right\rangle \cdot\left\langle I_{S G}, S_{-} G N E F\right\rangle+\left\langle i_{s g}, s g\right\rangle\left(c_{-} r a s\right) . \\
& \left\langle i_{c r}, \overline{c_{-} r a s}\right\rangle(g t p) \cdot\left\langle I_{S G}, S_{-} G N E F\right\rangle
\end{aligned}
$$

The following interactions are possible:

$$
\begin{array}{r}
\left\langle I_{I S I}, I N A S W I_{-} I\right\rangle \mid\left\langle I_{S G}, S_{-} G N E F\right\rangle \longrightarrow \\
\left\langle I_{A S I}, A C T S W I_{-} I\right\rangle \mid\left\langle I_{S G}, S_{-} G N E F\right\rangle \\
\left\langle I_{I S I I}, I N A S W I_{-} I I\right\rangle \mid\left\langle I_{S G}, S_{-} G N E F\right\rangle \longrightarrow \\
\left\langle i_{b}, b b o n e\right\rangle\left\langle I_{A S I I}, A C T S W I_{-} I I\right\rangle \mid\left\langle I_{S G}, S_{-} G N E F\right\rangle
\end{array}
$$

The interaction (9) shows that the domain $I N A S W I_{-} I$ of Ras is activated by the domain of $S_{-} G N E F$ of SOS. The interaction (10) shows that the domain $I N A S W I_{-} I I$ of Ras is activated by the domain $S_{-} G N E F$ of SOS.

The detailed Ipi programs for activated domains, ACTSWI_I, ACTSWI_II of the protein Ras and the domain $R_{-} N t$ of Raf are defined in (11) through (13):

$$
\begin{aligned}
\left\langle I_{A S I}, A C T S W I_{-} I\right\rangle::= & \left\langle i_{s}, \bar{s}\right\rangle\left(r s_{-} 2\right) \cdot\left\langle i_{i s 2}, \overline{r s_{-}}\right\rangle \cdot\left\langle I_{A S I}, A C T S W I_{-} I\right\rangle \\
& +\left\langle i_{b}, \overline{b b o n e}\right\rangle \cdot\left\langle I_{I S I}, I N A S W I_{-} I\right\rangle \\
\left\langle I_{A S I I}, A C T S W I_{-} I I\right\rangle::= & \left\langle i_{s g}, \overline{\text { sg }}\right\rangle\left(r_{-} s w i_{-} 1\right) \cdot\left\langle i_{r s 1}, r_{-} s w i_{-} 1\right\rangle(x) . \\
& \left\langle i_{b}, \overline{b b o n e}\right\rangle\left\langle I_{A S I I}, A C T S W I_{-} I I\right\rangle \\
\left\langle I_{R N}, R_{-} N t\right\rangle::= & \left\langle i_{s}, \bar{s}\right\rangle\left(c_{-} \text {ras }\right) .\left\langle i_{c r}, c_{-} \text {ras }\right\rangle \cdot\left\langle I_{A R N}, A C T R_{-} N t\right\rangle
\end{aligned}
$$

The processes so defined have the following interactions:

$$
\begin{array}{r}
\left\langle I_{I S I}, A C T S W I_{-} I\right\rangle \mid\left\langle I_{R N}, R_{-} N t\right\rangle \longrightarrow^{*} \\
\left\langle I_{I S I}, A C T S W I_{-} I\right\rangle \mid\left\langle I_{A R N}, A C T R_{-} N t\right\rangle \\
\left\langle I_{I S I I}, A C T S W I_{-} I I\right\rangle \mid\left\langle I_{G}, G A P\right\rangle \longrightarrow^{*} \\
\left\langle i_{b}, b b o n e\right\rangle\left\langle I_{I S I I}, I N A S W I_{-} I I\right\rangle \mid\left\langle I_{G}, G A P\right\rangle \\
\left\langle i_{b}, \overline{b b o n e}\right\rangle \cdot\left\langle I_{A S I I}, A C T S W I_{-} I I\right\rangle \mid\left\langle I_{A S I}, A C T S W I_{-} I\right\rangle \longrightarrow \\
\left\langle I_{I S I I}, I N A S W I_{-} I I\right\rangle \mid\left\langle I_{I S I}, I N A S W I_{-} I\right\rangle
\end{array}
$$

The interaction (14) shows that the active domain $A C T S W I_{-} I$ of Ras interacts with the domain $R_{-} N t$ of Raf. (15) shows that GAP inactivates the domain ACTSWI_II of Ras. (16) says that the domains of Ras interact with each other and that Ras rollbacks to the initial inactivated state.

When Ras mutates aberrantly, it does not have any effect on the Ras's binding with GTP and GDP but will reduce the activity of the GTP hydrolase of Ras and lower its hydrolysis of GTP greatly; in the meantime Ras will be kept in 
an active state; they keep activating the molecule, induce the continual effect of signal transduction, and result in cell proliferation and tumor malignancy.

(17) defines the Ipi representation of GAP in the aberrant state. (18) shows that GAP loses its function and does nothing, meaning that it can not inactivate the domain $A C T S W I_{-} I I$ of Ras.

$$
\begin{aligned}
& \left\langle I_{G}, G A P\right\rangle::=\langle 0, \S\rangle\left\langle i_{s}, s g\right\rangle\left(c_{-} r a s\right) \cdot\left\langle i_{c}, \overline{c_{-} r a s}\right\rangle(g d p) \cdot\left\langle I_{G}, G A P\right\rangle \\
& \left\langle I_{G}, G A P\right\rangle \longrightarrow\langle\emptyset, 0\rangle
\end{aligned}
$$

But then the interaction (16) will not occur whereas the interaction (14) will occur infinitely. Now observe that

$$
\langle 0, \sharp\rangle\left\langle I_{A S I}, A C T S W I_{-} I\right\rangle \longrightarrow\left\langle I_{A S I}, A C T S W I_{-} I\right\rangle ;\langle 0, \sharp\rangle\left\langle I_{A S I}, A C T S W I_{-} I\right\rangle
$$

It reaches in an abnormal state with exceptions. The pi calculus could not easily describe this aberrant case. Ipi calculus, on the contrary, can describe it quite precisely. In fact, when the aberrance occurs, it will be marked into the tag. So we can check the existing aberrance, using the tag system of Ipi calculus.

\section{Future Prospects}

The Ipi calculus is an extension of the pi calculus. The desirable outcomes and properties of a biochemical process can be formally proven in the framework of the Ipi caluclus. The theory of process calculus allows us to formally compare two programs using bisimulation. We can also define different levels of bisimilarity for verifying different properties of biochemical processes.

The research opens up new possibilities in the study of biochemical systems with exceptions. Designing and implementing an automatic tool will be our next work.

\section{References}

1. Milner, R., Parrow, J., Walker, D.: A Calculus of Mobile Processes, parts I and II. In: Information and Computation. (1992) 1-77

2. Priami, C., Regev, A., Silverman, W., and Shapiro,E.: Application of a stochastic name passing calculus to representation and simulation of molecular processes. In: Information Processing Letters. 80(2001) 25-31

3. Regev, A.: Representation and simulation of molecular pathways in the stochastic pi calculus. In: Proceedings of the 2nd workshop on Computation of Biochemical Pathways and Genetic Networks. (2001)

4. Regev, A., Silverman, W., and Shapiro, E.: Representing biomolecular processes with computer process algebra: pi calculus programs of signal transduction pathways. In: http://www.wisdom.weizmann.ac.il/ aviv/papers.htm (2000)

5. Regev, A., Silverman, W., and Shapiro, E.: Representation and simulation of biochemical processes using the pi calculus process algebra. In: Proceedings of the Pacific Symposium of Biocomputing. 6(2001) 459-470

6. Sangiorgi, D., and Walker, D.: The pi calculus: a Theory of Mobile Process. In: Cambridge University Press. (2001) 